\title{
Growth and characteristics of type-II InAs/GaSb superlattice-based detectors
}

\author{
A. Khoshakhlagh*, D. Z. Ting, A. Soibel, L. Höglund, J. Nguyen, S. A. Keo, A. Liao, and S. D. \\ Gunapala
}

Jet Propulsion Laboratory, California Institute of Technology

\begin{abstract}
:
We report on growth and device performance of infrared photodetectors based on type II InAs/Ga(In)Sb strain layer superlattices (SLs) using the complementary barrier infrared detector (CBIRD) design. The unipolar barriers on either side of the absorber in the CBIRD design in combination with the type-II InAs/GaSb superlattice material system are expected to outperform traditional III-V LWIR imaging technologies and offer significant advantages over the conventional II-VI material based FPAs. The innovative design of CBIRDS, low defect density material growth, and robust fabrication processes have resulted in the development of high performance long wave infrared (LWIR) focal plane arrays at JPL.
\end{abstract}

Keywords: Unipolar barrier, infrared detectors, superlattice, long-wave

\section{Introduction:}

The type-II InAs/GaSb superlattice (SL) system has been investigated as a promising system for infrared (IR) detection ever since it was proposed by Smith and Mailhiot [1] over three decades ago. In SL structures, the energy difference between the electron miniband and the first heavy-hole state at the Brillouin zone center defines the electronic bandgap; therefore the bandgap can be tailored by varying the thickness of the two constituent materials. Thus, the type-II InAs/GaSb SL cut-off wavelength can be tuned to span a broad spectrum from mid-infrared (MIR) to very long-infrared (VLIR) $(3 \mu \mathrm{m}<\lambda<30 \mu \mathrm{m})$ by changing the InAs and GaSb layer thicknesses. In this material system, due to the type II band alignment (see figure 1 [2]), the electron and hole wavefunctions are localized in the InAs and GaSb layers, respectively. In such a configuration, the two wavefunctions overlap better in thin-layer SL structures, resulting in larger absorption coefficients. As the overlap between the wavefunctions is strongest near the hetero-interfaces the optical absorption is restricted to the vicinity of interfaces [3].Thus, one can conclude that by atomic engineering the superlattice interfaces and by changing the thicknesses of InAs and $\mathrm{GaSb}$, the absorber cutoff wavelength can be tuned from 3 to more than $30 \mu \mathrm{m}$ while maintaining high quantum efficiency.

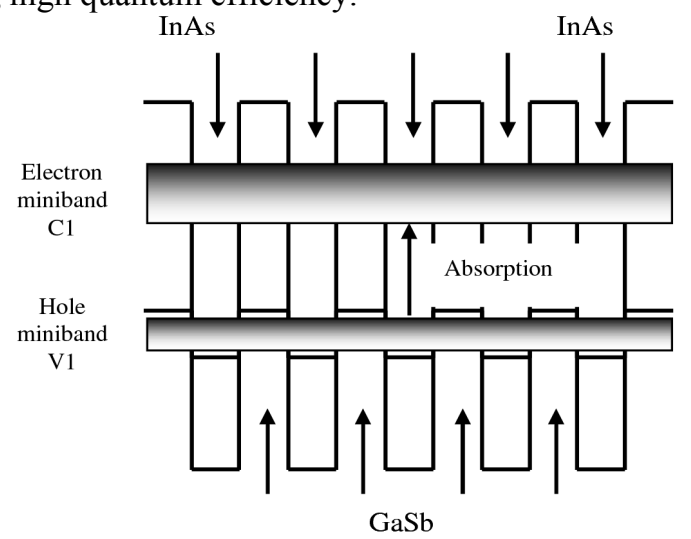

Figure 1: Band edge diagram illustrating the confined electron and hole minibands which form the energy band gap.

Infrared Remote Sensing and Instrumentation XIX, edited by Marija Strojnik, Gonzalo Paez, Proc. of SPIE Vol. 8154, 81540K · ( 2011 SPIE · CCC code: 0277-786X/11/\$18 - doi: 10.1117/12.894286 
Furthermore, the extra degrees of freedom in designing the bandstructure of the SL can be explored to enhance the performance of the detectors fabricated from such an absorber material. As an example, tunneling currents in SLs are reduced due to a larger electron effective mass. Also, large splitting between heavy-hole and light-hole valence subbands due to strain in the SLs contributes to the suppression of Auger recombination. Moreover, the band structure of the SLs can be engineered to enhance carrier lifetimes [4] and reduce noise at higher temperatures [5]. SL based IR detectors have demonstrated high quantum efficiency [6], high-temperature operation [7] and are suitable for incorporation in focal plane arrays by tapping into the mature III-V based growth [8] and fabrication processes [9].

Despite the above advantages there still exists challenges with type-II InAs/GaSb SL devices regarding the suppression of band-to-band and defect-assisted tunneling currents as well as surface leakage currents. More recently, barrier infrared detector (BIRD) concepts such as the $\mathrm{nBn}$ [10] device design have shown promising results to be superior to superlattices in the MWIR region. We have demonstrated a new design, so-called the complementary barrier infrared detector (CBIRD) [11] which enables high LWIR performance. The CBIRD design (shown in figure 2) incorporates two unipolar barriers - one on each side of the superlattice absorber layer.

Here we demonstrate that high performance focal plane arrays (FPAs) can be developed based on the CBIRD architecture, utilizing the antimonide materials system grown on GaSb substrates.

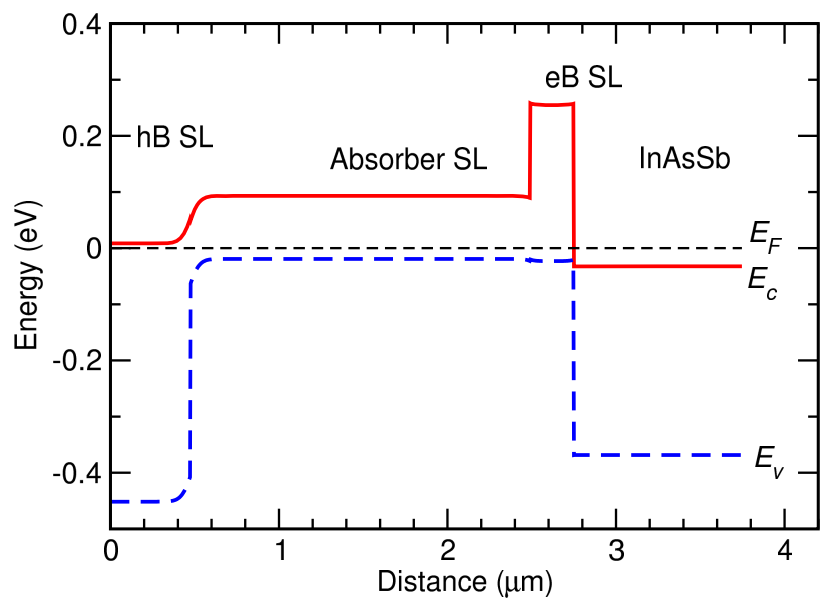

Figure 2: The energy band diagram of the complementary barrier infrared detector structure, showing the conduction and valenceband edges and the Fermi level under zero bias.

\section{Device Growth and Material Characterization}

CBIRD SL photodiodes were grown in a Veeco Applied-Epi Gen III molecular beam epitaxy chamber equipped with valved cracking sources for the group V Sb2 and As2 fluxes. Growth was performed on Te-doped n-type GaSb (100) substrates. During growth substrates were separated from the molybdenum wafer holders by a $50 \mathrm{~mm}$ outer-diameter pyrolytic boron nitride ring in front and a sapphire backing plate in back. Indium and gallium growth rates were determined by monitoring intensity oscillations in the reflected high-energy electron diffraction (RHEED) patterns. The LWIR CBIRD superlattice device structure has been published elsewhere [11].

Figure 3 shows the XRD of the LWIR CBIRD structure. Three different sets of satellite peaks are representative of the three different types of SLs used in the structure. The most pronounced satellite peaks originate from the thick absorber region, with the second tallest peaks coming from the top AlSb/InAs hole barrier, and the weakest peaks coming from the buried, thinner MWIR electron barrier. Intense satellite peaks with a FWHM of the zero-order peak equal to 16 arcsec for the absorbing region is attesting to the good crystalline quality of the layers and the high reproducibility rate in the SL period. 


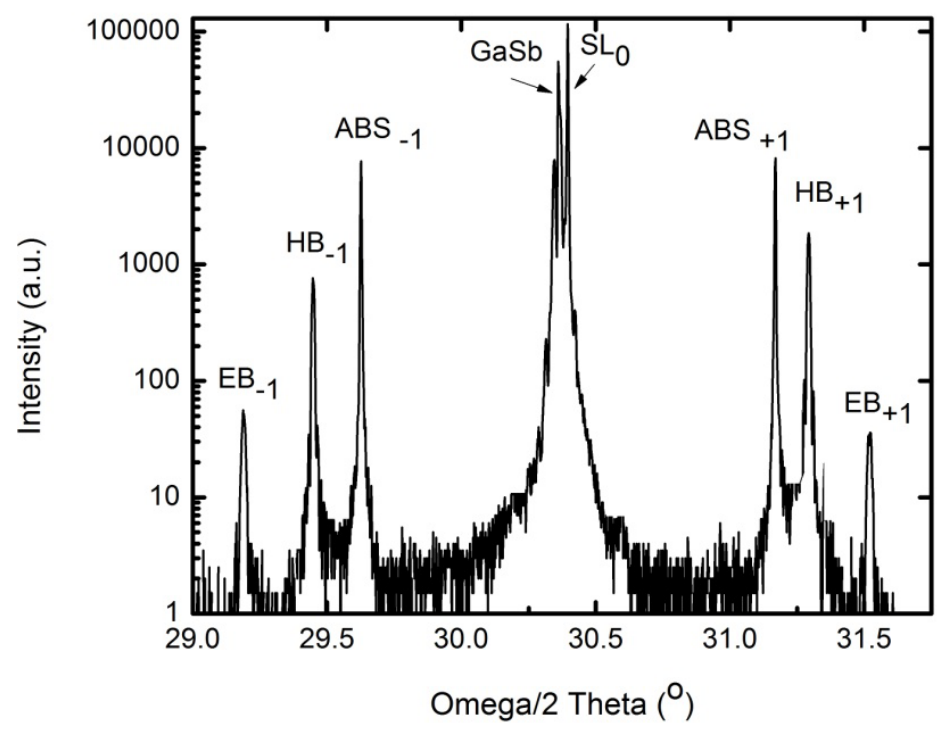

Figure 3: XRD scan of the CBIRD structure near the GaSb (004) reflection, showing three distinct superlattices: the absorber (ABS), the hole barrier $(\mathrm{HB})$ and the electron barrier (EB).

Atomic force microscope (AFM) scans of the surface of the as-grown epitaxial CBIRD structure, shows very smooth surface, and typically no large defects are visible on an $80 \mu \mathrm{m} \times 80 \mu \mathrm{m}$ scan (not shown) of the wafer surface. Higherresolution $20 \mu \mathrm{m} \times 20 \mu \mathrm{m}$ scans (figure 4 ) of the wafer surface are very smooth, and the image contrast comes mainly from monolayer steps on the surface. From these measurements a mean surface roughness of 2 to $4 \AA$ can be extracted. Surfscan measurements with a Tencor 6220 light point defect analysis generally reveals a density of less than $200 / \mathrm{cm}^{2}$ of micron or larger sized defects. This density of large defects is entirely within the acceptable range for this type of epitaxial growth, and does not preclude the material from being suitable for FPA applications.

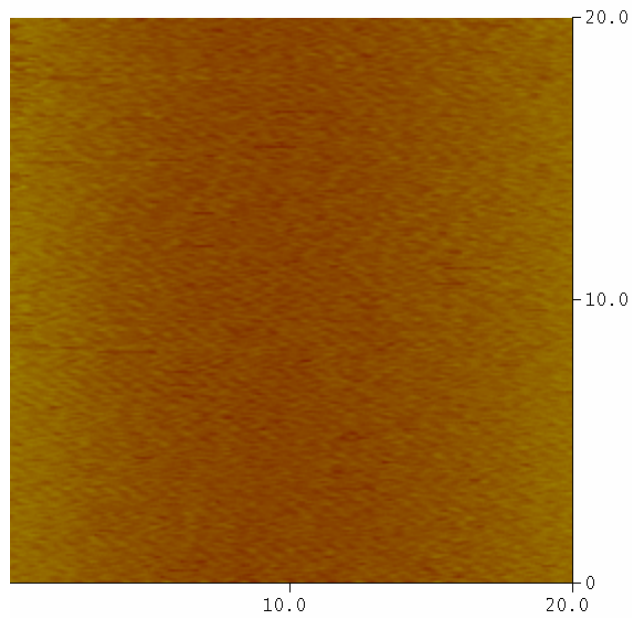

Figure 4: $20 \mu \mathrm{m} \times 20 \mu \mathrm{m}$ AFM scan of the CBIRD structure.

PL measurements were performed using a Thermo-Fisher Fourier transform infrared (FTIR) spectrometer operated in the step-scan mode. The samples were mounted on the cold-finger in a continuous flow cryostat. A $658 \mathrm{~nm}$ laser diode was used as excitation source and the photoluminescence signal was measured with cooled a HgCdTe detector. The 
measured PL spectrum shows a clear peak centered at $10 \mu \mathrm{m}$ with FWHM of $18.7 \mathrm{meV}$. The high PL intensity and narrow FWHM indicate a high crystalline quality of the material.

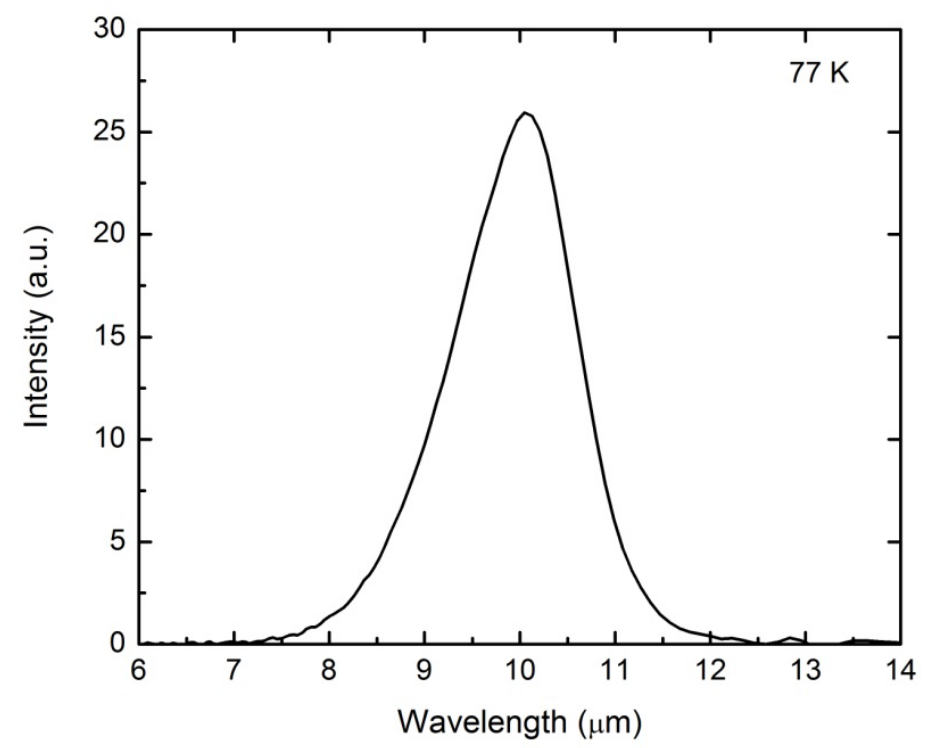

Figure 5: PL of the CBIRD structure at $77 \mathrm{~K}$.

\section{Device Characterization}

Single element detectors with square mesas of area $200 \times 200 \mu \mathrm{m}^{2}$ were fabricated using standard optical lithography, wet chemical etching and evaporation of top and bottom Ti/Pt/Au ohmic contacts. Current voltage $(I-V)$ characteristics of SL detectors were measured at $77 \mathrm{~K}$. As figure 6 indicates the current density is less than $j<1 \times 10^{-5} \mathrm{~A} / \mathrm{cm}^{2}$ at applied biases up to $V b=0.18 \mathrm{~V}$.

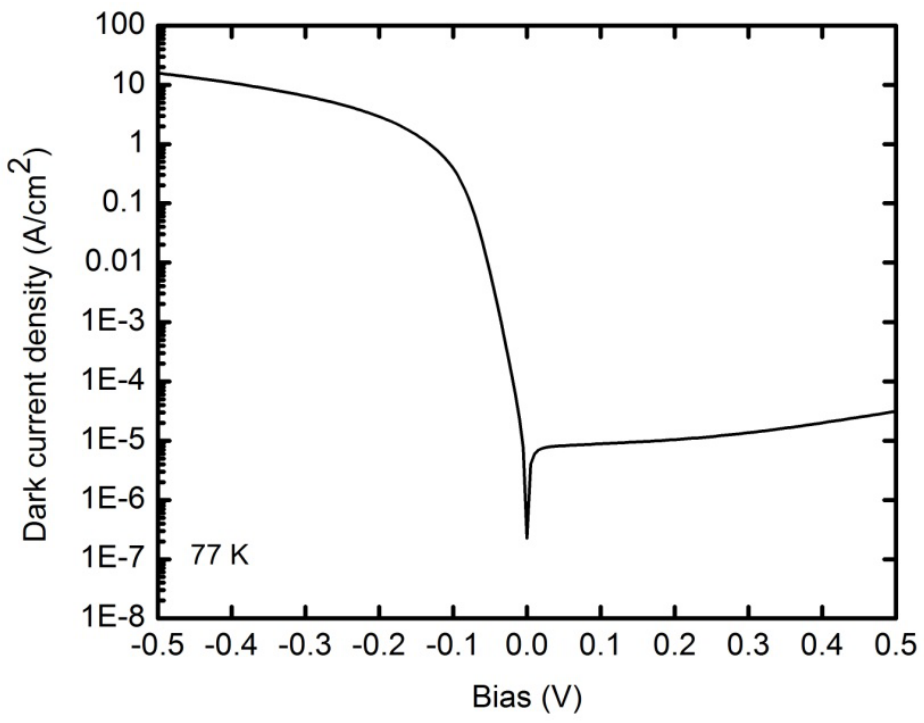

Figure 6: Dark current density of the LWIR CBIRD structure at $77 \mathrm{~K}$. 


\section{Imaging results}

We have fabricated a $320 \times 256$ format FPA using developed n-CBIRD SL wafers. Figure 7 shows images taken with this FPA at an operating temperature of 78K. Preliminary analysis indicates an operability of $98 \%$, and an NE $\Delta \mathrm{T}$ of $26 \mathrm{mK}$ with $300 \mathrm{~K}$ background.

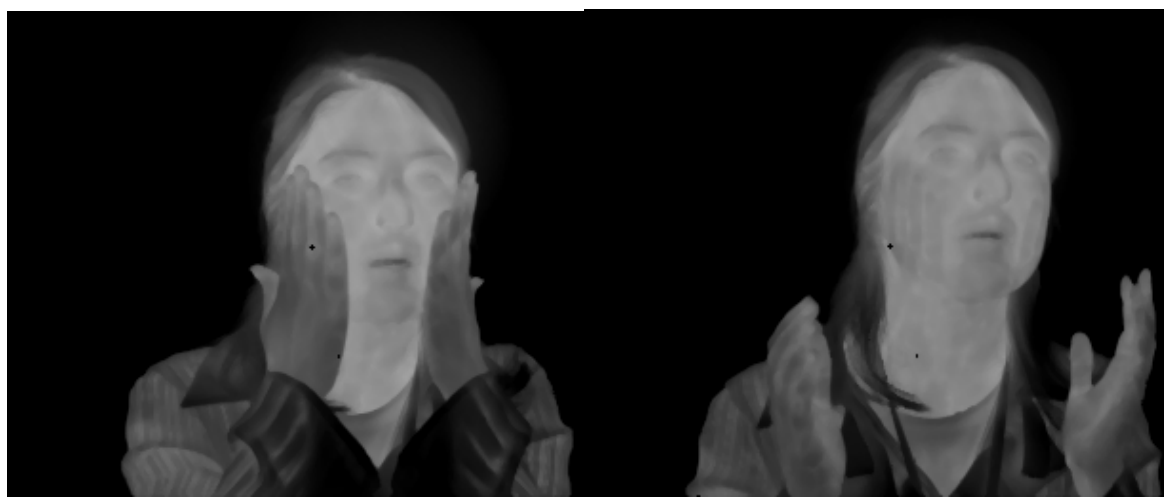

Figure 7: Images taken by the LWIR SLS based FPA (77 K).

\section{Conclusion}

Infrared detectors based on type-II InAs/GaSb SLs have shown high quantum efficiency and lower dark current as compared to other competitive technologies promising a viable platform for future MWIR and LWIR focal planar arrays. Complementary barrier design has theoretically shown reduction in band to band tunneling, generation-recombination and diffusion dark currents. The flexibility of the SL materials system as well as the elegant design of barrier infrared detectors (BIRDs) offer great potential both for higher-operating temperature and low background applications. In this work, long-wave SL detectors based on the CBIRD design were grown, and characterized. AFM, XRD, PL and surfscan measurements revealed high quality of the material grown at JPL. A 320x256 focal plane array, which were fabricated using developed wafers, demonstrated excellent performance with NE $\Delta \mathrm{T}$ of $26 \mathrm{mK}$ and $50 \%$ cutoff wavelength of $10 \mu \mathrm{m}$.

\section{Acknowledgments}

The authors thank C. J. Hill for helpful discussions, and M. Tidrow, R. Liang, M. Herman and E. Kolawa for encouragement and support. The research described in this publication was carried out at the Jet Propulsion Laboratory, California Institute of Technology, under a contract with the National Aeronautics and Space Administration.

\section{References}

[1] D.L. Smith, C. Mailhiot, J. Appl. Phys. 62 (1987) 2545.

[2] A. Rogalski and P. Martyniuk, Infrared Physics \& Technology. 48, 39 (2006).

[3] M. R. Kitchin and M. Jaros, Physica E: Low-dimensional Systems and Nanostructures. 18, (2003).

[4] C.H. Grein, P.M. Young, H. Ehrenreich, Appl. Phys. Lett. 61, 2905 (1992).

[5] E.R. Youngdale, J.R. Meyer, C.A. Hoffman, F.J. Bartoli, C.H. Grein, P.M. Young, H. Ehrenreich, Appl. Phys. Lett. 64, 3160 (1994). 
[6] R. Rehm, M. Walther, J. Schmitz, J. Fleibner, S. Kopta, F. Fuchs, W. Cabanski, J. Ziegler, J. Crystal Growth. 278, 1560161 (2005).

[7] A.Khoshakhlagh, E. Plis, S. Myers, Y.D. Sharma, L.R. Dawson, S. Krishna, Journal of Crystal Growth, 311,1901 (2009).

[8] J. Nguyen; A. Soibel; D. Z. Ting, C. J. Hill, M. Lee, S. D. Gunapala, Applied Physics Letters , 97, 051108 (2010).

[9] P.-Y. Delaunay, B.-M. Nguyen, D. Hoffman, M. Razeghi, IEEE J. Quantum Electron. 44462 (2008).

[10] S. Maimon and G. W. Wicks, Appl. Phys. Lett. 89, 151109 (2006).

[11] D.Z.Y. Ting, C. J. Hill, A Soibel, S.A. Keo, J.M. Mumolo, J. Nguyen, and S.D. Gunapala, Appl. Phys. Lett, 85, 023508 (2009). 